\title{
Gestión directiva universitaria desde el paradigma de la complejidad: otra mirada y nuevas propuestas ${ }^{*}$
}

\author{
FABER ANDRÉS ALZATE ORTIZ** \\ LUZ MERY CHAVERRA RODRÍGUEZ*** \\ ELVIA PATRICIA ARANGO ZULETA****
}

Recibido: 11 de junio de 2019 - Evaluado: 24 de noviembre de 2019 - Aceptado: 3 de diciembre de 2019

Citar como: Alzate Ortiz, F. A., Chaverra Rodríguez, L. M. y Arango Zuleta, E. P. (2020). Gestión directiva universitaria desde el paradigma de la complejidad: otra mirada y nuevas propuestas. Hallazgos, 17(33), 23-51. DoI: https://doi.org/10.15332/2422409X.5454

* Artículo derivado del proyecto de investigación interinstitucional denominado: "Los principios del paradigma emergente en las prácticas de gestión de los actuales directivos docentes universitarios: caso Universidad Católica de Oriente y Corporación Universitaria Minuto de Dios, Uniminuto".

** Doctor en Educación. Decano de la Facultad de Educación de la Corporación Universitaria Minuto de Dios (Uniminuto), Bello (Antioquia, Colombia).

Correo electrónico: faber.alzate@uniminuto.edu ORCID: https://orcid.org/0000-0002-3102-7666

*** Doctora en Educación. Docente investigadora de la Universidad Católica de Oriente (UCO), Colombia.

Correo electrónico: lchaverra@uco.edu.co ORCID: https://orcid.org/0000-0001-8979-3278

**** Doctoranda en Filosofía. Docente investigadora de la Universidad Católica de Oriente (UCO).

Correo electrónico: earango@uco.edu.co

ORCID: https://orcid.org/0000-0002-5930-0898 


\section{Resumen}

Este artículo se deriva de un proyecto de investigación interinstitucional cuyo objetivo central fue reconocer los principios del paradigma de la complejidad en las prácticas de gestión de los actuales directivos docentes universitarios. Dicho trabajo se desarrolló mediante un enfoque metodológico cualitativo, por tratarse de un estudio orientado a develar asuntos relacionados con la identificación de prácticas de gestión de los directivos docentes, la caracterización de los principios que están implícitos en dichas prácticas y la emergencia de posibles estilos de gestión a la luz del paradigma de la complejidad, como asuntos contextuales de la realidad universitaria en dos instituciones de educación superior ubicadas en Antioquia, Colombia. Entre las conclusiones más representativas se encontró que los principios de incertidumbre, autorregulación, autonomía/dependencia y recursividad son los más recurrentes en el quehacer de los actuales directivos universitarios. Estos representan, para la gestión de las instituciones estudiadas, una oportunidad para repensar creativamente las maneras como se vienen consolidando los proyectos, programas y planes de docencia, investigación y extensión, de suerte que puedan ser valoradas otras formas de liderar, y con ello de gestionar el conocimiento y las comunidades de aprendizaje que se han constituido dentro de su cotidianidad organizacional.

Palabras clave: calidad educativa, complejidad, educación, gestión directiva, mejoramiento, universidad. 


\title{
University directive management from the complexity paradigm: another look and new proposals
}

\begin{abstract}
This article derives from an inter-institutional research project whose main objective was to recognize the principles of the complexity paradigm in management practices of current university presidents. This work was developed through a qualitative methodological approach, since it is a study aimed at revealing issues related to the identification of management practices of presidents, the characterization of the principles that are implicit in these practices and the emergence of possible management styles in the light of the complexity paradigm, as contextual issues of university reality in two higher education institutions located in Antioquia, Colombia. Among the most representative conclusions, it was found that the principles of uncertainty, self-regulation, autonomy/dependence and resourcefulness are the most recurrent in the works of current university presidents. These represent, for the management of the institutions studied, an opportunity to rethink creatively the ways in which teaching, research and extension projects, programs and plans are being consolidated, so that other ways of managing knowledge can be valued, and with it manage knowledge and learning communities that have been established within their organizational daily life.
\end{abstract}

Keywords: educational quality, complexity, education, directive management, improvement, university.

\section{Gestão diretiva universitária desde o paradigma da complexidade: outro olhar e novas propostas}

\section{Resumo}

Este artigo deriva-se de um projeto de pesquisa interinstitucional cujo objetivo central foi reconhecer os princípios do paradigma da complexidade nas práticas de gestão dos atuais diretivos docentes universitários. Este trabalho se desenvolveu mediante um enfoque metodológico qualitativo, por tratar-se de um estudo orientado a desvelar assuntos relacionados com a identificação de práticas de gestão dos diretivos docentes, a caracterização dos princípios que estão implícitos nestas práticas e o surgimento de possíveis estilos de gestão à luz do paradigma da complexidade, como assuntos contextuais da realidade universitária em duas instituições de educação superior localizadas em Antioquia, Colômbia. Entre as conclusões mais representativas encontrou-se que os princípios de incerteza, auto-regulação, autonomia/dependência e recursividade são os mais recorrentes no afazeres dos atuais gerentes universitários. Estes representam, para a gestão das instituições estudadas, uma oportunidade para repensar criativamente as maneiras como se vem consolidando os projetos, programas e planos de docência, pesquisa e extensão, de sorte que possam ser valorizadas outras formas de liderar e com isso de administrar o conhecimento e as comunidades de aprendizagem que se tem constituído dentro de sua cotidianidade organizacional.

Palavras-chave: qualidade educativa, complexidade, educação, gestão diretiva, melhoramento, universidade. 


\section{Introducción}

Analizar la gestión que deben realizar los actuales directivos docentes de las universidades no solo es una oportunidad para replantear los límites teóricos de la clásica administración y planeación educativa, sino que también es un reto complejo si se acepta que es necesario interconectar intencional y sistémicamente los diferentes know how 'saber cómo' inherentes a cada líder e institución a la hora de pensar y actuar con relación a determinadas actividades que constituyen su cotidianidad, pero también su planeación estratégica a largo plazo. Dicha planeación, muy seguramente, en algunos asuntos, resulta similar para todas las universidades en temas como la gestión de personal calificado, la renovación de registros, las acreditaciones de calidad, la investigación, la proyección social, entre otros. Sin embargo, estos temas suelen ser abordados y tratados de manera diferente. Algunos dirían que esto es gracias a la autonomía universitaria ${ }^{1}$, pero otros considerarían que puede ser más por los efectos directos de la cultura organizacional que caracteriza a cada institución, producto de su experiencia acumulada, el tipo de personas que coexisten allí y las apuestas teleológicas que emanan de su proyecto educativo, dos realidades que son parte de las discusiones abordadas en este artículo.

No obstante, Martínez (2000) sitúa todo lo anterior en clave de lo que él denomina la cotidianidad de la gestión universitaria, la cual debe estar basada en

[...] diseñar nuevas formas de articulación con el resto de la sociedad, sumarse como protagonista a la revolución científica y tecnológica en curso y contribuir a alcanzar los objetivos de educación de calidad para todos, equidad, competitividad e innovación permanente, sin renunciar por ello al ejercicio de su función crítica. Para ello debe adecuar sus estructuras y modos de funcionamiento, por lo que la gestión universitaria es un elemento estratégico para superar tales retos. (p. 6)

Estos asuntos soportan las pretensiones académicas que se tuvo con la investigación "Los principios del paradigma emergente en las prácticas de gestión de los actuales directivos docentes universitarios: caso Universidad Católica de Oriente y Corporación Universitaria Minuto de Dios, Uniminuto, sede Bello”, de la cual deriva este artículo. En este documento se tomó como base problematizadora las diferentes situaciones de tensión en el nivel directivo, vividas a diario en estas dos instituciones,

1 La Constitución Política de Colombia, a través de su artículo 69, garantiza la autonomía universitaria. 
y que deben sortearse de forma estratégica y operativa constantemente por sus líderes. De esta forma, ellos deben garantizar, intrínsecamente, la subsistencia académica de los programas y la estabilidad administrativo-financiera de la institución $y$, extrínsecamente, el cumplimiento con el compromiso ético y político que poseen como institución de educación superior de brindar a la sociedad procesos de docencia, investigación y extensión que las transformen y que mejoren la calidad de vida de sus ciudadanos.

Aunque las actuales dinámicas académicas y administrativas de las universidades son tema de conversación inagotable en diferentes esferas de la vida académica, política y social del país y de las regiones, tras varios rastreos bibliográficos, se pudo determinar que a la fecha han sido pocas las investigaciones, tanto local como internacionalmente, en las que se identifican las prácticas de gestión que caracterizan el nivel directivo de las universidades. Estos hallazgos serían útiles para, quizá, documentar, analizar y comprender aquellas investigaciones que puedan considerarse un referente de buena práctica, de tal suerte que sean consultadas y aplicadas por otras instituciones. Con ello se podría consolidar un marco de referencia que congregue posibles corrientes de conocimiento universitario en el orden regional o hasta nacional, que transforme la actual manera de gestionar estas organizaciones.

Para esta investigación, se tomó como base epistemológica el paradigma de la complejidad. Con base en este se pretendió hacer lectura interrelacionada de las diferentes realidades de directivos universitarios y sus equipos de trabajo, para develar las maneras en que se percibían en la institución y cómo sorteaban en su día a día las diferentes situaciones que laboralmente debían afrontar, muchas de estas derivadas de sus planeaciones o programaciones, otras tantas emergentes no planeadas y en ocasiones ni sospechadas, pero que al final también se convierten en asuntos que dan sentido a su gestión como directivos universitarios.

\section{Marco de referencia}

\section{A manera de antecedentes}

Luego de revisar diferentes investigaciones relacionadas con las prácticas de gestión de directivos universitarios, se pudo constatar que aún son insuficientes — en relación con el número de trabajos desarrollados en otros campos de conocimiento afines- los desarrollos que existen sobre gestión directiva universitaria y sus saberes relacionales. Este hecho supone un campo de discusión aún fértil e inagotado que da 
mérito a la necesidad de caracterizar el rol de quienes hoy cumplen labores directivas y de gestión en el marco de las funciones sustantivas universitarias, con el propósito de comprender de fondo los retos que ciertamente tiene la universidad con el desarrollo de las comunidades donde hacen presencia con su proyecto educativo (Mentado y Rodríguez, 2011). Ahora, aunque son pocos los estudios existentes en este campo, como ya se mencionó, es posible develar tres grandes líneas teóricas sobre las cuales han versado los trabajos encontrados: la primera asume la universidad como una organización inteligente; la segunda considera que la universidad es una organización atípica y compleja, y la tercera supone que la gestión universitaria deberá estar orientada a la búsqueda de la excelencia.

Algunos estudios en la línea de la universidad como una organización inteligente (Senge, 1993; De Donini y Donini, 2003; Fallas, 2010, Castells, 2003; Zawacky-Richter, 2009) sugieren que la universidad de hoy no puede seguir siendo una organización que piense y actúe como en el pasado; por el contrario, está invitada a dar respuestas coherentes con las necesidades y potencialidades que poseen las comunidades actuales. En este contexto, la universidad debe estar atenta a los acelerados cambios que experimenta una región y un país, producto de las dinámicas tecnológicas y económicas de la globalización, lo cual le sugiere la generación de modelos de gestión más flexibles y dinámicos que impulsen estratégicamente su quehacer cotidiano, de manera que el aprendizaje y el diálogo sean sus pilares fundamentales.

Otros autores, como Mintzberg (1991), Mena (2004), García, Hernández, Santos y Fabila (2009) y Duque (2009), consideran que las dinámicas cotidianas en las que se mueven hoy las universidades en función de sus procesos y de su quehacer diverso hacen que esta institución sea una organización atípica, en la que sin duda resulta complejo la consolidación de un modelo de gestión educativo capaz de tener en cuenta las condiciones particulares de sus colaboradores y equipos, al tiempo que se lideran las pretensiones generales como forma de organizar y combinar recursos para alcanzar las metas.

Y otros autores, un poco más orientados hacia una gestión educativa universitaria centrada en los procesos que faciliten la búsqueda de la excelencia (Palacios, 1996; Kells, 1997; Rebolloso, Fernández, Pozo y Cantón, 2001; Tejedor, citado en Martínez, 2000; Pérez y Requena, 2000; Casassus, 2000), consideran que las universidades, como todas las instituciones formadoras, tienen una misión que cumplir, que en el imaginario colectivo están orientadas hacia la prestación de un servicio educativo de calidad. Esto le exige a sus directivos no solo contar con un adecuado paquete de 
retos estratégicos que dirijan en el tiempo su desarrollo e indiquen el sentido de su orientación hacia el logro, sino que además antepone como requisito que todos sus líderes sean capaces de tomar decisiones pertinentes para gestionar los procesos y recursos necesarios que les permitan materializar los objetivos universitarios. Por esta razón, estos directivos deben reconocer permanentemente qué tan bien están ejecutando sus labores, con el propósito de ajustar aquellos aspectos que necesitan ser perfeccionados, de manera que puedan desempeñarse de forma eficaz y eficiente en el marco de la mejora continua.

Lo anterior sugiere un panorama que permite suponer que la gestión directiva universitaria puede estar mayormente orientada hacia una plataforma teóricopráctica basada en principios de linealidad, certeza y orden. Esta es una realidad que no significa que se esté actuando mal, pero que leída en tiempo presente puede no ser suficiente para atender estratégicamente el volumen de actividades que se deben desarrollar en la actual carrera por la acreditación de alta calidad, la renovación de registros calificados, las certificaciones internacionales, los ranking nacionales e internacionales, la formación de alto nivel del profesorado, el bienestar institucional, la internacionalización del currículo, entre otros tantos asuntos, que en la actualidad forman parte de la larga lista de compromisos que deben liderar los directivos universitarios, y para lo cual un pensar y hacer lineal y dicotómico no resulta precisamente el más efectivo.

Según algunos antecedentes de Bonnefoy, Cerda, Peine, Durán y Ponce (2004),

[...] las universidades nacionales e internacionales están llevando a cabo evaluaciones de la calidad de sus procesos, entre los cuales se cuentan: exámenes acuciosos de sus mallas curriculares, procesos de autoevaluación y evaluación de pares externos, del mismo modo, comienza a institucionalizarse la necesidad de acreditar las carreras, programas de formación inicial o de post-grado; como también examinar rigurosamente la calidad de los profesionales que egresan de sus aulas. (p. 79)

Estas tareas, según Bonnefoy et ál. (2004), están llevando a mecanizar la cotidianidad de estas instituciones, creando así unas angustias que no son precisamente las del escenario académico, las cuales por décadas se consideraron el foco de su propia gestión. No obstante, sumado a todas las actividades ya indicadas — para muchos, exceso de burocracia y modas banales de la época-, hay quienes piensan que las apuestas de gestión deben ir más allá y delimitan una serie de responsabilidades más atractivas y retadoras en el campo universitario, con lo cual dejan ver que puede 
ser posible construir un proyecto educativo universitario centrado en las relaciones, las oportunidades, la innovación y el dinamismo.

Por ejemplo, Elizondo (2011) considera que algunas de las responsabilidades centrales de quienes en la actualidad dirigen el rumbo de las universidades son:

1. Crear y construir una visión y misión compartida con todos los involucrados en el proceso educativo, en particular con los directores y profesores.

2. Fomentar tenazmente el trabajo colaborativo, a través de la formación de grupos de trabajo que participen en cada una de las responsabilidades y proyectos mencionados.

3. Crear y construir el sentido de pertenencia, para disminuir la resistencia al cambio y fomentar la adopción de los proyectos, a través de hacer copartícipes a los participantes del proceso educativo.

4. Promover el trabajo interdisciplinario, de vital importancia, a través de la creación de redes cara-a-cara o medios electrónicos entre los miembros de las diferentes carreras, ya que una buena parte de las funciones se trabajan de esta manera.

5. Liderar, facilitar el cambio y lanzar iniciativas para promover cambios educativos.

6. Desarrollar los hábitos indispensables para un buen directivo, en especial la autoconciencia, la empatía, la retroalimentación con arte, el establecimiento de límites apropiados y la flexibilidad con las personas.

7. Práctica de la paciencia, tolerancia, respeto, capacidad de apertura y negociación.

8. Compromiso, entrega y pasión, elementos indispensables que hacen que las cosas sucedan.

9. Determinación, disciplina, esfuerzo y responsabilidad propia hacia toda acción.

10. Búsqueda y acción incansable para hacer realidad el sueño. (pp. 215-216)

De tales responsabilidades, leídas en clave de las diferentes necesidades que tienen actualmente las universidades, es importante mencionar por lo menos tres retos:

1. Mayores niveles de articulación interinstitucional con el propósito de impulsar procesos de docencia, investigación y extensión que respondan a estándares de alta calidad a nivel tanto nacional como internacional. 
2. Profesionales cualificados asignados a cada una de las funciones sustantivas, es decir, personal altamente capacitado para desarrollar sus funciones y trabajar en equipo.

3. Más y mejores procesos de gestión que soporten integralmente el quehacer de las unidades o equipos desde una visión sistémica y autoorganizada.

Como puede leerse, las exigencias y los desafíos son para quienes constituyen los cuerpos de trabajo académico dentro de las universidades.

\title{
Una mirada desde la teoría como punto de partida epistémico
}

\section{Hacia un pensar complejo en la vida universitaria}

\author{
Todo fenómeno debe ser considerado en su ecosistema: el \\ pensamiento complejo ha exigido considerar el pensamiento como \\ ecologizado en su relación coorganizadora con su ambiente.
} BARBEROUSSE (2008, p. 104)

Como ya se ha destacado en párrafos anteriores, se ha tomado como referente teórico el paradigma del pensamiento complejo:

Mientras que el pensamiento simplificador desintegra la complejidad de lo real, el pensamiento complejo integra lo más posible los modos simplificadores de pensar, pero rechaza las consecuencias mutilantes, reduccionistas, unidimensionalizantes y finalmente cegadoras de una simplificación que se toma por reflejo de aquello que hubiere de real en la realidad. (Morin, 2009, p. 22)

Dicho paradigma propone, para este caso, redimensionar las dinámicas mismas del pensar y hacer universitario, de manera que sea posible considerar de forma holística otros principios de actuación directiva no exactamente tan lineales, lo que abre paso a nuevas formas de desarrollar la gestión académico-administrativa en el corto, mediano y largo plazo.

Ahora bien, Roger-Ciurana (2001-2002) afirma que una forma compleja de pensar pone en marcha una serie de principios de pensamiento que pueden servir como guía de la acción: 
- Principio de construcción de movimiento: puesta en marcha de un pensamiento en bucle, que va del todo a las partes y de las partes al todo. Del análisis a la síntesis, y de la síntesis al análisis.

- Principio de bucle retroactivo: ruptura de la causalidad lineal.

- Principio de recursividad: un bucle recursivo es aquel cuyos productos son necesarios para la continuación del proceso. Esta es la base de la autoorganización y de la autoproducción.

- Principio dialógico: llamamos "dialógico" a aquel proceso relacional en el que, en un mismo espacio, concurren de forma complementaria y antagonista, lógicas diferentes. Fomentar la dialógica no es tanto fomentar el desorden, como fomentar la creatividad.

- Principio de integración del sujeto en la observación y el conocimiento.

- Principio de autonomía/dependencia: toda organización necesita de una apertura relativa del sistema y de un relativo cerramiento.

- Principio hologramático: los individuos reflejamos la sociedad, al igual que la parte refleja la totalidad del holograma. (pp. 16-17)

Estos siete principios pueden ser considerados columnas vertebrales del pensar complejo universitario, claves para que sus directivos se puedan ver avocados a construir otros caminos de posibilidad en su gestión, de manera que integren intencionalmente su saber, ser y hacer profesional con el horizonte teleológico de la institución y las exigencias no menores del entorno. Sin embargo, según Elizalde (2013):

El pensamiento complejo entraña un modo de aproximación al mundo y al ser humano, que rechaza cualquier intento de interpretación simplificada y reduccionista.

[...] En su apuesta antropocéntrica, el pensamiento complejo entraña una invitación a situarse ante el mundo con una actitud diferente, a fin de captar la realidad como verdaderamente es, asumiéndola con pertinencia. (p. 48)

Estos tres escenarios conceptuales - los de Morin, Ciurana y Elizalde — tienen una línea epistemológica común que concibe el pensamiento complejo como la oportunidad para ampliar el espectro de las clásicas formas de generar, transformar y utilizar el conocimiento y las verdades científicas tradicionalmente constituidas. De este modo, promueven la emergencia de otras racionalidades capaces de interactuar desde el plano de la inter y la transdisciplinariedad para crear nuevos/otros modelos/ 
referentes de gestión y privilegiar otras prácticas directivas, y también para soportar la lectura y la comprensión que en la actualidad se puede hacer de la gestión que los directivos universitarios están desarrollando cotidianamente al tratar de cumplir los propósitos teleológicos que emanan de los proyectos educativos universitarios (PEU) en una era de crisis, incertidumbre y parametrización.

Efectivamente, leer desde la perspectiva de un pensar complejo todas y cada una de las diferentes situaciones en las que se ven implicados los directivos universitarios puede resultar, desde una mirada epistémica, una oportunidad para pasar de una visión centrada en la teoría o en un plan prescriptivo a una basada en la acción estratégica y creativa y en un hacer con sentido, en el que se privilegie un construir colectivo contextualizado, de manera que se genere aquello que Barberousse (2008) considera un pensamiento ecologizado.

En este sentido, es imposible considerar la vida universitaria como un sistema lineal o cerrado, en el que todo se puede y se debe explicar desde la posición de la causaefecto o desde la simplificación de una comunicación unidireccional y monológica. Por el contrario, la universidad y sus procesos son un sistema abierto y complejo de realidades que requieren un saber, ser y hacer dialógico, retroactivo, dinámico, integrador, hologramático y recursivo, en los que el centro de la acción siempre sea la persona y su entorno. Por lo tanto, lo complejo en la vida universitaria nunca será lo complicado, lo enredado o lo difícil de crear o decir. Lo complejo es la oportunidad que tiene el directivo universitario, y con él la comunidad en general, de liderar el rumbo de la institución, según los siguientes aspectos:

- La creación e inmersión en redes de aprendizaje y conocimiento con otros académicos y con la población a la cual debe su propósito misional.

- Transformar los acuerdos cerrados y lineales en cartas de navegación abiertas, críticas y multifacéticas, de forma que quepan todos los coequiperos y sus capacidades.

- Reconocer múltiples maneras de abordar y resolver las situaciones problema.

- Devolver la voz a los actores implicados y con ello su protagonismo en la construcción de la cultura organizacional.

- Promover una relación armónica entre sus miembros y el entorno, creando así, a manera de ecología cognitiva, otras maneras de percibir, abordar y sentirse en la real realidad.

- Cultivar una ética de la solidaridad y el respeto por la diferencia a partir de la cual se potencie la condición humana de sus colaboradores. 
- Impulsar una cultura de la sostenibilidad universitaria basada en un pensamiento ecologizado y holístico.

Estas pueden considerarse premisas que, en sintonía con los principios del pensamiento complejo y las raíces epistemológicas de este paradigma, sugieren generar nuevos metapuntos de referencia sobre la gestión universitaria, de tal suerte que faciliten reconsiderar el perfil mismo de sus directivos.

\section{Hacia una gestión directiva en renovación conceptual y práctica}

Como lo propone Villareal (2005), la gestión directiva es "el conjunto de acciones integradas para el logro de un objetivo a cierto plazo que comprende, entre otras, las administrativas, de personal, económicas, de planificación y de programación” (p. 1). Esto, en clave del actual desarrollo de las universidades, representa tres apuestas interdependientes que vale la pena destacar:

1. Una gestión directiva eficaz, adecuada y pertinente, con la cual sea posible movilizar los esfuerzos de la comunidad hacia la consecución de los objetivos institucionales.

2. Una gestión institucional que reconoce los procesos y está orientada hacia los resultados, lo que supone la consolidación de una cultura permanente de autoevaluación, coevaluación y heteroevaluación con miras al mejoramiento continuo.

3. Una gestión universitaria que reconoce la importancia de formar integralmente su talento humano con el propósito, por un lado, de prepararlo para los diferentes cambios que se afrontan en el ámbito educativo y, por el otro, para el uso estratégico del conocimiento, de manera que se logren formas más flexibles de organización en las que las jerarquías son definidas en función de la acumulación de capacidades y no de los cargos que ostentan las personas.

Lo anterior compromete en tiempo presente un tipo de liderazgo renovado y renovador en el contexto de las universidades, capaz de promover una cultura de la gestión del conocimiento, a partir de la cual sea posible volver explícitos los saberes tácitos de sus colaboradores, mejorar los conocimientos y las prácticas especializadas que se poseen y construir rutas de innovación que transformen la cultura académica y directiva organizacional (Farfán y Garzón, 2006; Alzate y Jaramillo, 2015).

En este sentido, la visión de los directivos universitarios deberá ser menos la de un clásico administrador y más la de un gestor educativo, de manera que se consoliden nuevas/otras miradas en torno a las personas y los equipos humanos que 
colaboran en la materialización del PEU y a la institución como planta física, con el fin de que se construya una visión holística y una mirada sustentable de todo lo que en sí constituye la vida universitaria. De esta forma se gesta una renovada escala de valores en la que lo principal sea la persona y el entorno, y desde allí partan todas las estrategias o acciones que se implementen a manera de un ecosistema universitario.

En este orden de ideas, son muchas las razones para postular la importancia de promover prácticas directivas diferenciales que permitan articular las funciones sustantivas, trabajar los planes estratégicos institucionales alineados con las experiencias y expectativas de los equipos de colaboradores y gestionar mejor el conocimiento, pues la gestión universitaria es

[...] percibida como los hechos llevados a cabo por los diferentes gestores que configuran el aparato organizativo de la universidad y que, a su vez, se distribuyen en varios niveles de la organización. Además, podemos hablar entonces de aquellas personas que ostentan la responsabilidad en la organización superior en la cota más alta del staff y que afectan al desarrollo de los distintos pilares de la universidad, docencia, investigación y gestión. (Rodríguez y Aguiar, 2015, p. 69)

Dentro de este contexto, se espera que los directivos universitarios sean capaces de comportarse idóneamente para pensar y liderar la estrategia institucional, al ser ellos quienes desde el nivel en el que se encuentren en la institución, están llamados a dinamizar el trabajo con otros colaboradores y lograr que todos caminen hacia un mismo norte. Este panorama permite exhortar a construir una cultura del trabajo en equipo que potencie las capacidades individuales y grupales, reconozca las limitaciones personales e institucionales e incorpore la escucha y la comunicación para el entendimiento, el liderazgo transformador, el respeto por la diferencia, entre otros atributos que superan la comprensión clásica de dirigir o liderar.

Lo anterior lleva a considerar que el desarrollo estratégico de las universidades en la actualidad requiere líderes capaces de pensar de forma compleja, sistémica, recursiva, holística y dinámica, que puedan gerenciar estratégicamente en sus prácticas cotidianas las situaciones de incertidumbre, caos, impotencia, resistencia al cambio, desmotivación, entre otras tantas situaciones que suelen ser las constantes en la actual vida universitaria.

En consonancia con lo dicho, hoy las universidades no pueden basar la totalidad de sus decisiones en actitudes que se lean en clave de certezas o verdades absolutas. Por el contrario, tienen que incluir en sus planes y programas espacio para el azar y para la no linealidad absoluta, teniendo en cuenta que las cosas no suceden 
simplemente porque así se considere, sino que es necesario tener presente otras variables como la madurez personal y profesional de los equipos, el lugar donde se pretende impactar, la tecnología, la pertinencia de los recursos que se poseen, la volatilidad y velocidad de la información, las capacidades financieras de operación, la experiencia acumulada para implementar algo y hasta la misma competencia que representan las otras universidades que se encuentran en el entorno, entre otras.

\section{Metodología}

El objeto de esta investigación fue reconocer los principios del paradigma de la complejidad en las prácticas de gestión de los actuales directivos docentes universitarios de Antioquia. Esto con el fin de mostrar una manera de pensar, con criterio de innovación y pertinencia, por un lado, los futuros y requeridos estilos de dirección y, por el otro, la manera como estos permitirían la transformación organizacional de las universidades en un momento histórico en el que los procesos de acreditación, los nuevos modos de investigar y de producción de conocimiento, la internacionalización de sus currículos, la gestión y el impacto social son variables que sugieren otras y nuevas formas de ser, actuar y pensar la vida universitaria.

El estudio fue de carácter cualitativo; se orientó hacia una investigación inductiva, a través de la cual se pretendió desarrollar comprensiones situadas y originales de la realidad de los actores implicados y los escenarios en los que se desenvuelven, desde una perspectiva holística y flexible que permitiera caracterizar las diferentes realidades universitarias en las que se ven inmersos sus directivos. De esta forma, se pretendió alcanzar una riqueza interpretativa y una contextualización pertinente. La población objeto de estudio formó parte de la Universidad Católica de Oriente y de la Corporación Universitaria Minuto de Dios (Uniminuto), sede Bello. La muestra estuvo conformada por veinte directivos docentes que llevaran por lo menos un año de experiencia en el cargo, que lideren determinado equipo de trabajo, y que además desarrollaran procesos de gestión académica y administrativa ${ }^{2}$.

2 La información sobre el enfoque, la determinación de la población y la muestra son datos extraídos textualmente del documento original del proyecto del cual deriva este artículo. Por lo tanto, algunos de los enunciados expuestos en el ítem de metodología podrán coincidir con otras publicaciones académicas, sin que ello signifique una mala práctica con respecto a los derechos de autor o desconocimiento alguno por parte de los autores del presente trabajo, que al mismo tiempo son los autores del proyecto de investigación. 
Al tratarse de un tema poco abordado, el método fue un estudio de casos. Esto permitió desarrollar el problema propuesto con precisión y minucia, con la intención de conocer de forma particular y detallada las vivencias narradas por los actores participantes (directivos y sus equipos de trabajo) desde su papel profesional. Así se pudieron consolidar paquetes de información enriquecidos por datos reales y casos cotidianos, que permitieron desde las diferencias caracterizar, según los principios del paradigma emergente, la realidad en la que se ve inmersa la gestión universitaria (Chaverra, Arango y Alzate, 2019).

Se diseñó una pauta de entrevista semiestructurada para directivos y otra para sus colaboradores (subordinados directos), para recolectar la información que después se procesó con el software Atlas Ti. De este modo se crearon redes semánticas con las categorías centrales derivadas de los diferentes principios del paradigma emergente, lo cual dio lugar a un análisis comprensivo de los datos finalmente obtenidos. Para llegar acá, fueron vividos tres momentos interdependientes, cada uno con actividades específicas y resultados esperados.

El momento 1 permitió la identificación de las prácticas de gestión de los directivos docentes. Para ello se seleccionaron aleatoria y voluntariamente colaboradores que trabajaran en las universidades vinculadas con la investigación y que cumplieran con los criterios de la muestra. Se les presentó el proyecto y se formalizó su participación voluntaria; se diseñaron y validaron los instrumentos a través de expertos y algunos pilotajes controlados $\mathrm{y}$, finalmente, se aplicaron las respectivas entrevistas semiestructuradas a cada uno de los directivos y a los miembros de su equipo de trabajo (subordinados directos).

En el momento 2, se procedió al reconocimiento de las características de los principios del paradigma de la complejidad implícitos en las prácticas de los directivos docentes. Aquí se partió del análisis de la información que se obtuvo en cada una de las entrevistas realizadas, las cuales fueron transcritas y procesadas en Atlas Ti. Simultáneamente se avanzó en la interpretación de los datos a través de un proceso de codificación, lo que dio como resultado unidades hermenéuticas que luego terminaron en cinco grandes redes semánticas. De estas, las dos primeras dan cuenta de los códigos extraídos de la entrevista con directivos; las dos siguientes, de los códigos extraídos de la entrevista con los colaboradores miembros de los equipos que lideran dichos directivos, y la última integra selectivamente todos estos códigos encontrados alrededor de los principios del paradigma de la complejidad, como radiografía final de la realidad de los procesos de gestión en la universidad. 
El momento 3 se dedicó a reconocer los principios del paradigma de la complejidad en las prácticas de gestión de los actuales directivos. Para esto se tomó como referente la última red semántica emergente que situó los principios más recurrentes y cada una de sus citas argumentativas, y así se elaboró una caracterización que permitió comprender, de manera más objetiva, los temas con mayor relevancia que pueden ser considerados en el marco de las actuales acciones directivas universitarias.

\section{Resultados}

\section{Momento 1: ¿Cómo ven los directivos docentes los procesos de gestión de la universidad?}

Los imaginarios que tienen los directivos universitarios de sus procesos de gestión articulados con las dinámicas propias de sus instituciones muestran que existen infinidad de posturas, no siempre muy comunes, en torno a la manera de afrontar las diferentes situaciones que se presentan en su día a día. En las figuras 1 y 2 se pueden observar algunas de las posturas que este grupo de profesionales tiene en relación con los procesos de gestión que deben desarrollar.

Como se puede analizar en las anteriores redes, las diversas posiciones develan unas características especiales en la dinámica del actual directivo universitario que vale la pena resaltar:

- Aunque el cambio es una constante en la vida de las universidades, no todos los líderes están preparados para enfrentarlo y gestionarlo adecuadamente.

- Es frecuente tener que resolver situaciones no previstas en la programación que se tiene debido a factores externos no controlados.

- Cuando hay colaboradores comprometidos es más fácil para el líder no solo enfrentar los desafíos de su gestión, sino también implementar acciones nuevas en pro del mejoramiento institucional.

- Aunque se espera que toda la gestión que realizan los directivos se enmarque en las políticas y lineamientos institucionales, en ocasiones hay situaciones emergentes que exigen actuar de forma flexible y dinámica.

- La gestión de un directivo nunca podrá leerse como una pieza aislada de las demás áreas; es necesario comprenderlas como acciones interdependientes que suman para el alcance de los objetivos institucionales.

- Es importante que los directivos no solo reconozcan las capacidades y experticias de sus coequiperos, sino también que sean capaces de aprovecharlas, de manera 


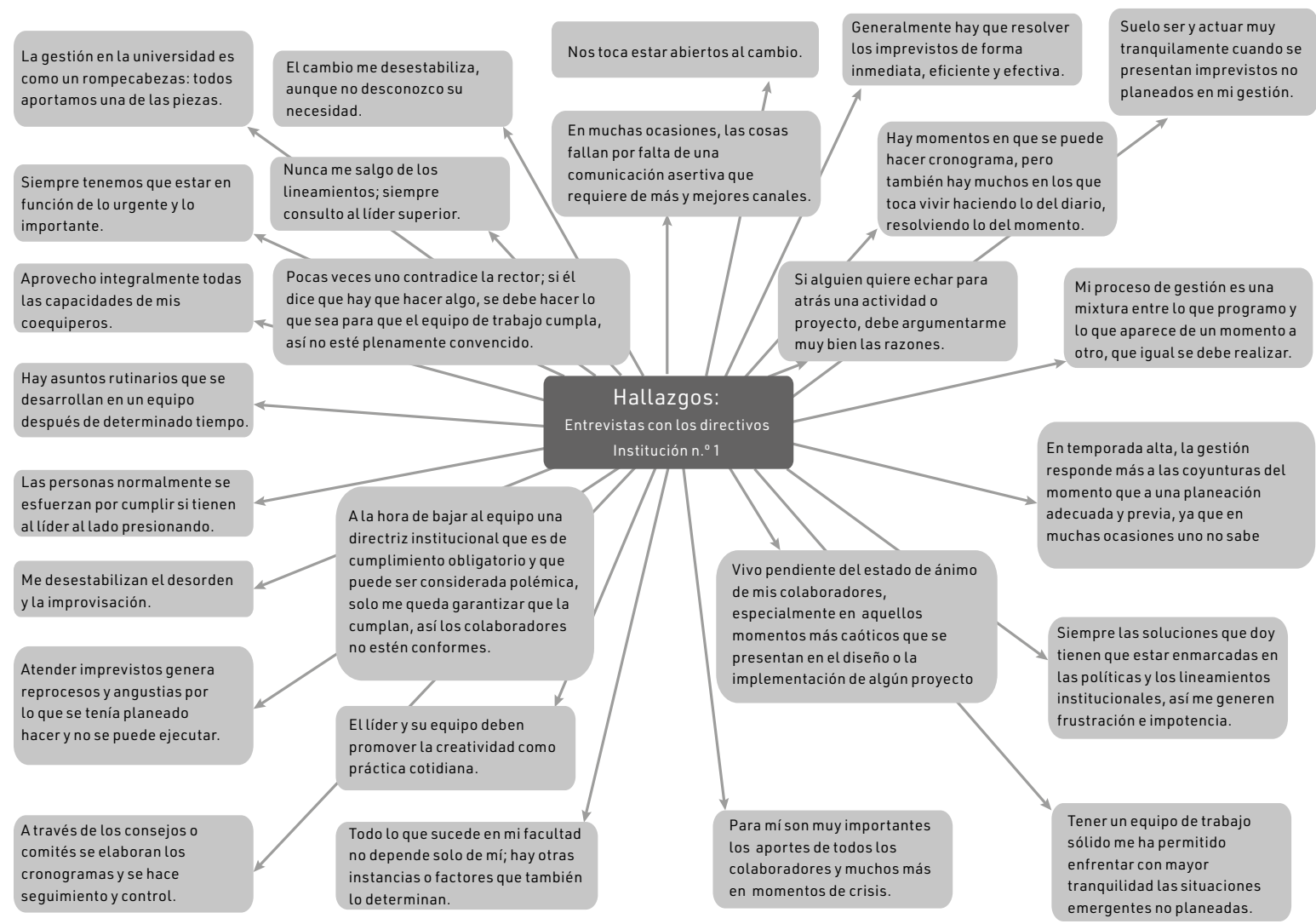

Figura 1. Red semántica de directivos de la Institución de Educación Superior n. ${ }^{\circ} 1$

Fuente: elaboración propia 


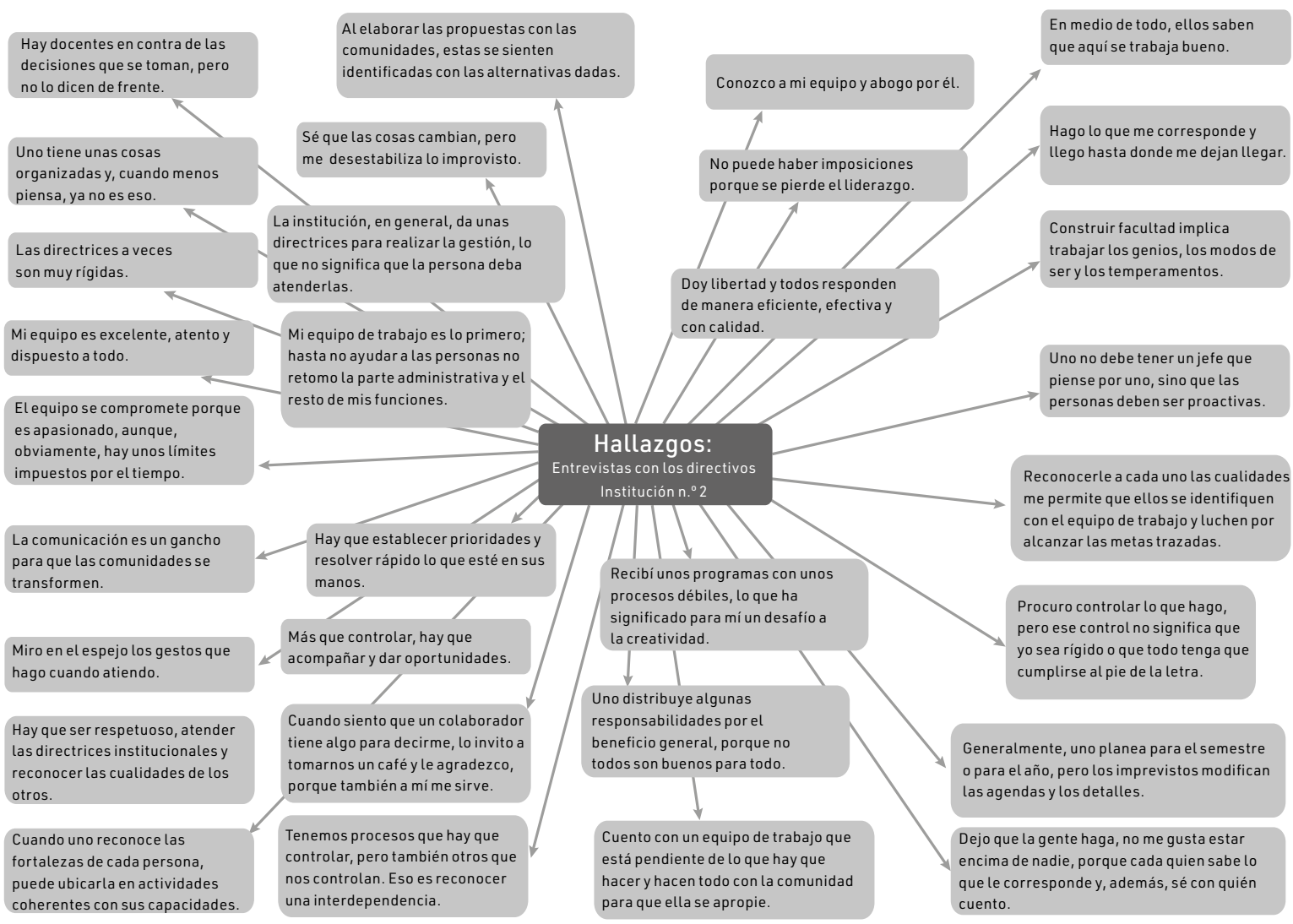

Figura 2. Red semántica de directivos de la Institución de Educación Superior n.ํํㄹ 2

Fuente: elaboración propia 
que se hagan más dinámicas y potentes las formas de trabajar en la formulación o puesta en marcha de un proyecto o actividad que se debe desarrollar.

- Aunque no existen planes de acciones que permitan atender de manera estratégica y organizada las diferentes situaciones de tensión que se presentan en aquellas temporadas o momentos más álgidos que se pueden vivir en la institución, lo que se hace normalmente es echar mano de todo aquello que permita actuar de forma adecuada para responder a la coyuntura del momento.

- En ocasiones es necesario imponer ciertas directrices, aunque los colaboradores no se encuentren muy de acuerdo, ya sea porque vienen dadas por una instancia institucional superior (consejos de gobierno, rectoría o vicerrectorías), o porque así lo determina la ley en el plano nacional o regional.

- Los procesos de gestión del actual directivo universitario son una mixtura amorfa, ya que están condicionados no solo por las planeaciones/programaciones que se elaboran de manera consciente con los equipos de trabajo, y que son valoradas y aprobadas por las instancias superiores, sino que, además, están supeditados a las diferentes actividades/tareas, compromisos que aparecen de forma imprevista y que tienen, en muchos casos, carácter de urgente.

- En procura de mantener unido y comprometido al equipo de trabajo, los directivos deben ingeniárselas para motivarlos y hacerlos sentir bien con las actividades que desarrollan, especialmente en aquellos momentos en que se presentan grandes volúmenes de trabajo, aparecen tareas no planeadas o hay que cumplir directrices que no son del todo cómodas y que desestabilizan al colaborador.

- Un directivo universitario debe ser capaz no solo de ser, sino también de promover con sus coequiperos altos niveles de creatividad, innovación, solidaridad, dinamismo, invención, imaginación e ingenio.

\section{Momento 2: ¿Cómo ven los miembros de los equipos los procesos de gestión de su líder?}

Al conversar con los colaboradores que apoyan la gestión del directivo universitario, es posible develar asuntos comunes relacionados con las percepciones y acciones del mismo líder. Pero también emergen otros aspectos que ponen de manifiesto la necesidad de consolidar prácticas de gestión más democráticas, pertinentes y modernas, que tengan como fin único no solo en el resultado sino también en el proceso, pero que además se basen en el dominio de una inteligencia emocional lo suficientemente desarrollada que permita ayudar a los equipos a afrontar los momentos/situaciones de crisis, de tensión o de satisfacción. Como se observa en las figuras 3 y 4, son varios los aspectos que 


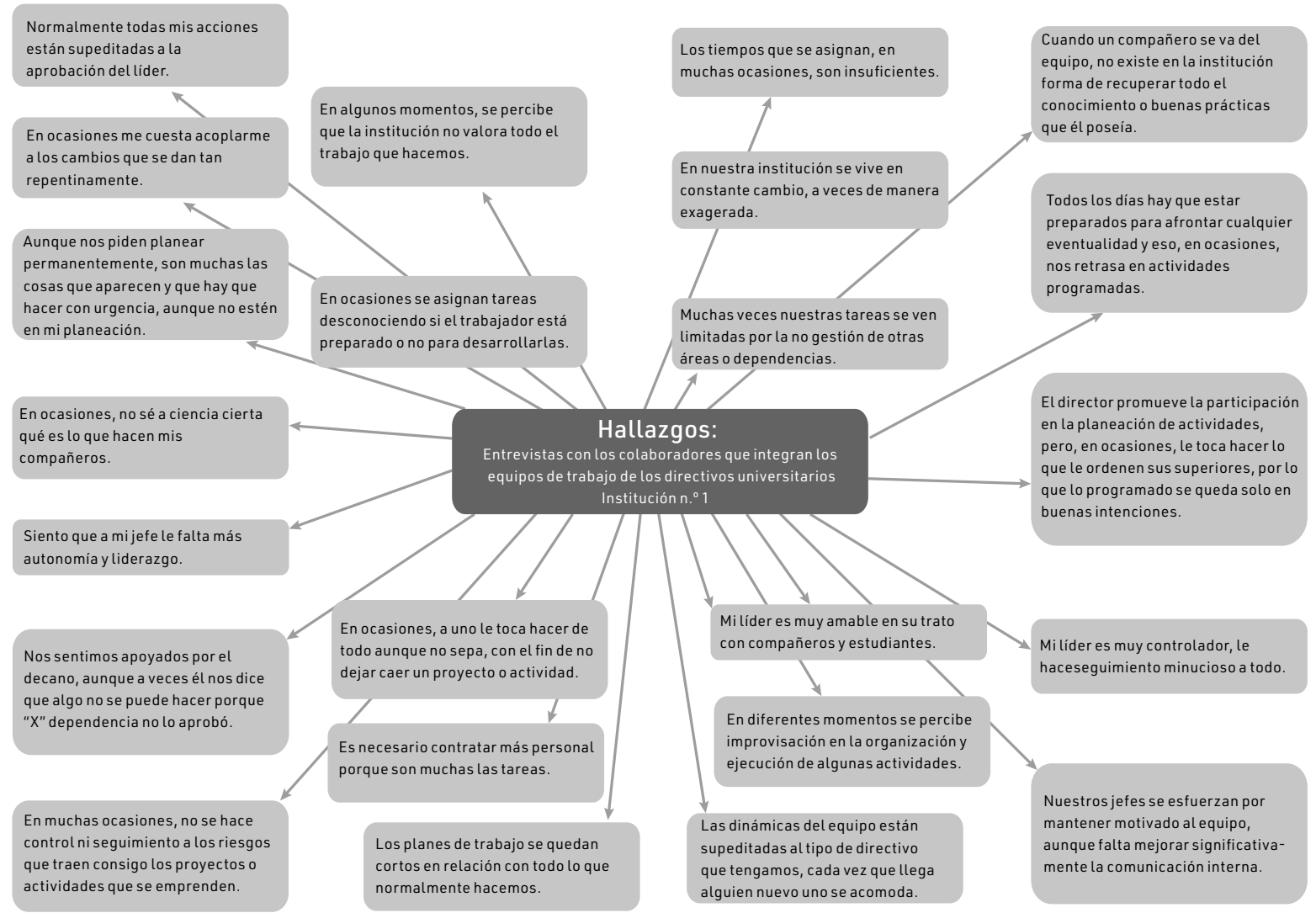

Figura 3. Red semántica de los coequiperos de los directivos de la Institución de Educación Superior n. ${ }^{0} 1$ Fuente: elaboración propia 


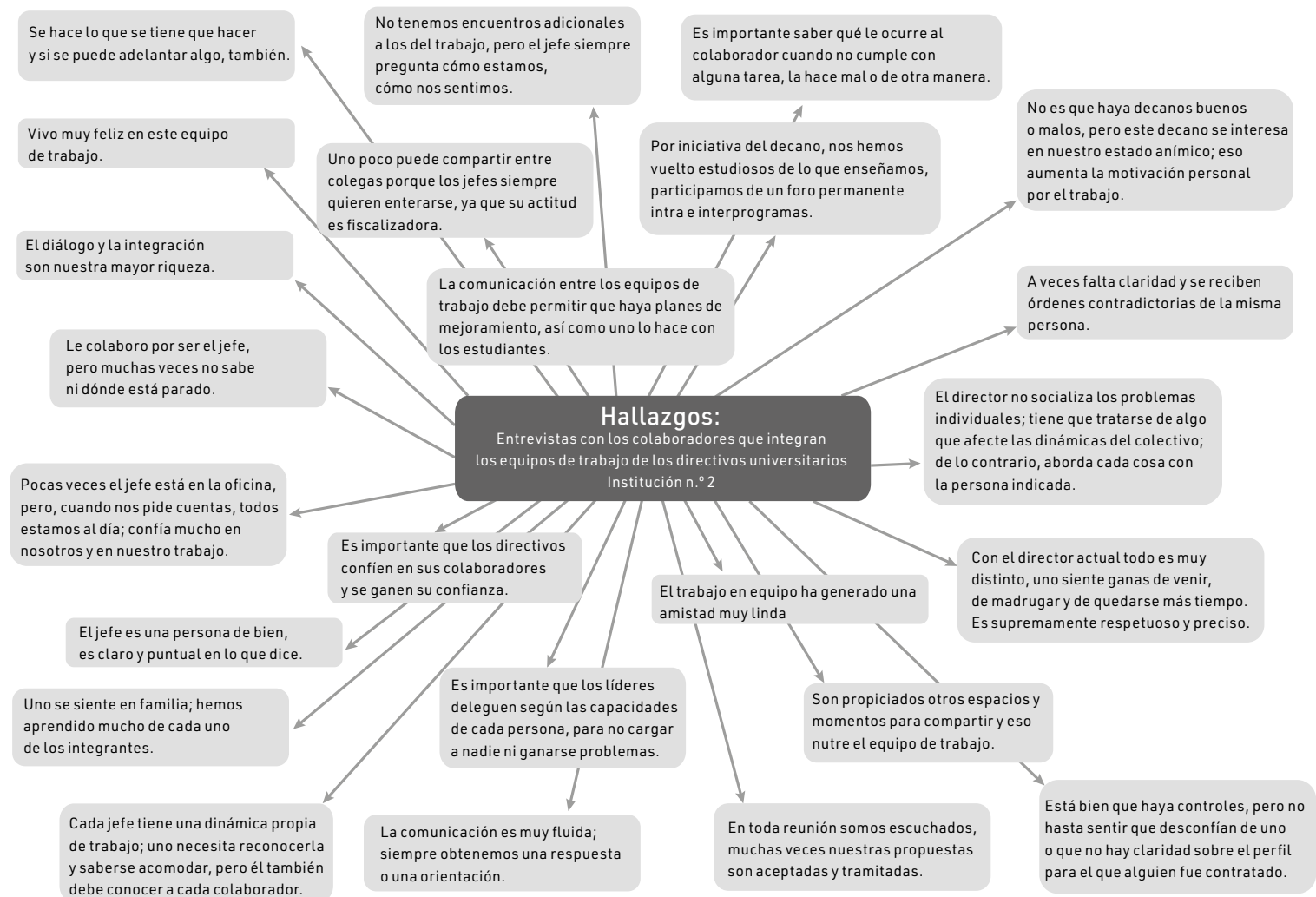

Figura 4. Red semántica de los coequiperos de los directivos de la Institución de Educación Superior n.ํํㄹ 2

Fuente: elaboración propia 
resaltan los colaboradores a la hora de valorar las prácticas y experiencias asociadas a las dinámicas institucionales.

De estas redes semánticas emergen algunos asuntos que pueden ser tenidos en cuenta estratégicamente por los directivos universitarios para potenciar su labor, y con ello favorecer el buen funcionamiento de las áreas a su cargo al tiempo que le suman al alcance de los objetivos institucionales:

- Es importante reconocer no solo los perfiles sino también la experiencia acumulada que posee un colaborador del equipo, de manera que las actividades que le sean asignadas, por un lado, puedan llegar a ser de su total agrado y, por el otro, sean adecuadas y pertinentes para lograr ser eficiente y efectivo.

- El proceso de acompañamiento que se hace al equipo debe superar la mirada clásica del control y la medición del resultado, incorporándole otras acciones como la motivación al logro, el empoderamiento, la identificación de alertas tempranas, la implicación en las actividades, el respaldo ante situaciones conflictivas, la tolerancia al fracaso, la gestión del riesgo y la auto y coevaluación periódica.

- Es indispensable poseer o generar canales de comunicación sencillos pero eficientes, a través de los cuales todos los colaboradores puedan tener acceso a la información que les permita desarrollar mejor sus labores.

- En el momento de diseñar e implementar un proyecto o actividad es necesario definir muy bien las áreas que estarían involucradas de forma directa o indirecta, hacerlas partícipes del plan de trabajo y garantizar que cumplan con sus compromisos, de manera que se pueda llevar a cabo una gestión sincronizada en la cual la labor de cada colaborador no se vea limitada sino potenciada.

- Lograr que los colaboradores alcancen un alto nivel de empoderamiento puede minimizar la excesiva dependencia y aumentar el nivel de productividad y motivación en el desarrollo de las actividades asignadas.

- Como directivos, es necesario hacer una adecuada asignación de los tiempos que se le deben dar a un colaborador para emprender una tarea, teniendo en cuenta no solo la etapa de diseño, sino también la de ejecución y la de evaluación; hay que procurar no saturar y de ser necesario garantizar el número de personas necesarias para que se pueda cumplir con las metas propuestas.

- Es fundamental que los miembros de un equipo reconozcan a su jefe como un líder, que vean en él las competencias para guiarlos y que genere la confianza suficiente a la hora de emprender cualquier labor. Ahora, no se pretende que se las sepa todas, pero sí que tenga la capacidad de brindar posibles salidas o recurrir a otras instancias que faciliten el soporte que se requiere. 


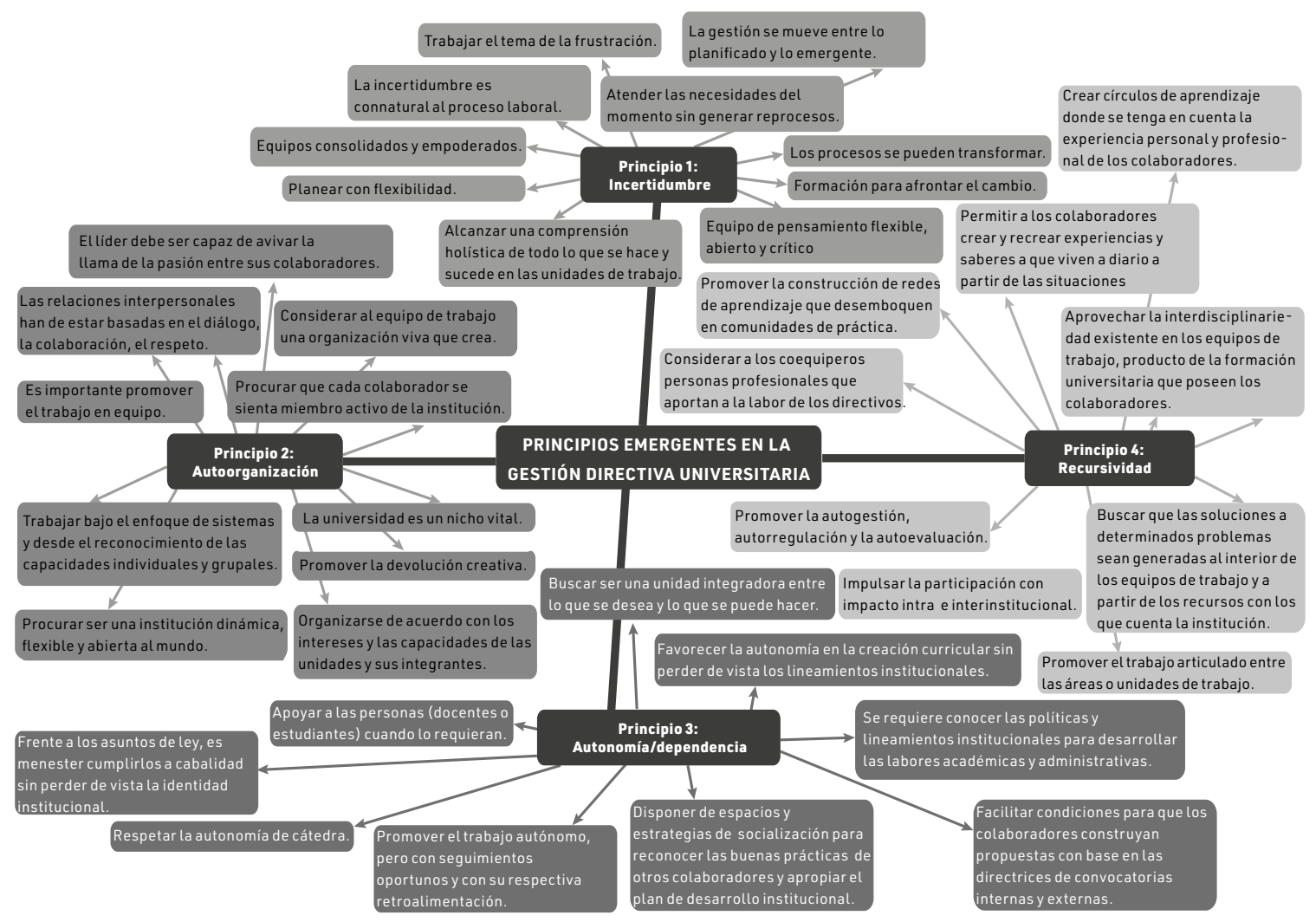

Figura 5. Red semántica de los principios del pensamiento complejo emergentes

Fuente: elaboración propia 


\section{Principios emergentes en las prácticas de gestión de los directivos universitarios}

Tomando como referente el escenario epistemológico del paradigma del pensamiento complejo para cotejar cada una de las respuestas que se obtuvieron de los directivos y sus colaboradores, emergieron cuatro relaciones muy fuertes que mostraron una tendencia hacia cuatro principios de este paradigma: incertidumbre, recursividad, autonomía/dependencia y autoorganización. Es importante destacar que fue de los planteamientos de los actores-informantes que se extrajeron aquellas expresiones más representativas y, posteriormente, se agruparon teniendo en cuenta que compartieran una misma línea epistémica desde la intención o desde la acción, lo que dio emergencia a determinado principio, como se muestra en la figura 5.

\section{Momento 3}

Como se puede observar en la red semántica de la figura 5, aparece no solo una serie de prácticas directivas e institucionales asociadas a determinados principios, sino que además se alcanza a reconocer, a manera de sugerencias, algunos postulados que pueden ser tenidos en cuenta para potenciar el quehacer universitario en una época que reclama nuevas maneras de ser, conocer y hacer en el mundo educativo. A continuación, se describirán los cuatro principios con algunas generalizaciones que dan razón de los elementos teórico-prácticos que tuvieron emergencia con la investigación y que, por motivos de estructura y extensión del presente trabajo, se han resumido:

- Principio de incertidumbre. La vida universitaria está en constante cambio; nada puede concebirse $100 \%$ seguro ni predecible. Las personas deben tener una comprensión holística de todo lo que sucede y realiza la unidad o facultad de la que participan. Los procesos de planeación deben funcionar como sistemas flexibles y abiertos que además permitan empoderar a los colaboradores y formarlos para afrontar el cambio y la frustración.

- Principio de recursividad. Cada una de las áreas, unidades o facultades necesitan trabajar de manera articulada. Su engranaje debe permitir potenciar sus capacidades instaladas, en la medida en que se aprovechen estratégicamente las capacidades personales y profesionales de cada colaborador. La visión del actuar debe considerar prácticas no solo en el orden intrainstitucional, sino también en el interinstitucional, aprovechando así las potencialidades de otros equipos (externos a la institución) para crear más y mejores fuerzas de trabajo.

- Principio de autonomía/dependencia. Sin perder de vista las políticas y los lineamientos que emanan de los consejos de gobierno institucional o de las leyes o normativas municipales, departamentales o nacionales, es importante reconocer 
el valor de la autonomía para crear propuestas académicas que potencien la investigación, la docencia y la extensión, preferiblemente desde una visión integradora entre los deseos personales y profesionales y las viabilidades en el hacer. No obstante, aunque se promueva un pensar y actuar soportados en la autogestión, la autorregulación, el autocontrol, es necesario diseñar e implementar medidas de seguimiento y control basadas en un acompañamiento respetuoso, solidario, creativo, implicado, estimulante y permanente.

- Principio de autoorganización. La autoorganización permite considerar la institución como una organización viva. Por tanto, es dinámica, flexible, creativa e innovadora, y requiere la gestación de procesos académicos y administrativos en los cuales todos los actores se sientan implicados, convocados, cocreadores. Estas situaciones exigen un liderazgo transformador basado en la promoción de la participación, el respeto por la diferencia y la cultura del trabajo en equipo. En equipos autoorganizados, las relaciones de poder son de orden horizontal y el reconocimiento está dado por las capacidades que posee cada persona y no por la posición que ocupa/ostenta en la jerarquía organizacional. Se promueve un pensar-actuar crítico y se trabaja desde la inteligencia emocional.

\section{A manera de conclusión}

Según Martínez (2007), en un sistema "se da un conjunto de unidades interrelacionadas de tal manera que el comportamiento de cada parte depende del estado de todas las otras, pues todas se encuentran en una estructura que las interconecta" (p. 133). En este sentido, la universidad como sistema requiere pensar su gestión a partir de estrategias directivas que se soporten en el trabajo en equipo, la comunicación asertiva, el empoderamiento colectivo, la democratización del conocimiento, el respeto por las diferencias y la solidaridad académica y administrativa. Todo esto, sin duda, sugiere promover la consolidación de nuevos estilos directivos en los cuales los liderazgos se basen en la autoorganización, la autorregulación, la incertidumbre y la recursividad, como algunos de los pilares para impulsar una gestión universitaria renovada.

Lo anterior se soporta en los resultados obtenidos y permite situar las siguientes consideraciones que al final no son exclusivamente puntos de llegada, sino más bien de partida, para seguir pensando el sentido de la gestión en el contexto de las universidades colombianas y su proyecto socioacadémico de ofrecer educación de calidad. 
En primer lugar, es importante comprender que la gestión de los hoy directivos universitarios no solo podrá estar supeditada a la capacidad para planear, ejecutar y evaluar determinadas tareas, un asunto importante pero no exclusivo, sino que además es fundamental que posea o desarrolle la capacidad para reconocerse, potenciarse, orientarse, acompañarse y construirse recíprocamente con los otros durante el profesional ejercicio de su labor. Adviértase que no es pretensión prescindir de los recursos ni desconocer los procedimientos o políticas institucionales para la gestión, sino, como se logró develar en los resultados, es buscar la realización de cada una de esas acciones a partir del reconocimiento intersubjetivo de los otros, del contexto y de sí mismo, de manera que no se desnaturalice la labor directiva producto de las dificultades que suelen emerger de los "afanes" administrativos.

En segundo lugar, las universidades que profesan creer y vivir en la calidad como filosofía de desarrollo y competitividad organizacional tienen el compromiso inminente de repensarse a partir de una visión más compleja de la realidad, teniendo en cuenta que la incertidumbre es y será un asunto que estará presente en su diario vivir y que les exigirá superar las clásicas formas de planear su desarrollo. Además, tendrán que hacerse más conscientes de su condición de sistema vivo, por lo que la autoorganización supondrá un reto crítico con base en el cual se verían modificadas las históricas maneras de administrar, gerenciar y liderar la institución. Pero también será necesario potenciar todas las áreas de acuerdo con su articulación intencionada, de manera que situando el principio de recursividad sea posible consolidar un modelo de gestión del conocimiento basado en el aprendizaje colaborativo y el empoderamiento colectivo, que sin desconocer las normas y las políticas internacionales, nacionales, departamentales e institucionales, en el marco de la autonomía/dependencia, faciliten la consolidación de planes, proyectos y programas de docencia, investigación y extensión más innovadores y pertinentes con la filosofía de la universidad y el territorio.

Finalmente, los cambios a los que están abocadas las universidades en la actualidad tienen asidero fuerte en la comunicación y la creatividad, dado que: 1) expresar las políticas institucionales, los propósitos del trabajo, las funciones y los procedimientos que se deben seguir no es sinónimo de comprensión, entendimiento y compatibilidad con lo propuesto; 2) comprender a partir de la comunicación implica reconocer el contexto, los valores y los significados como elementos gruesos e ineludibles entre los actores; 3 ) la comunicación jerárquica puede ser funcional siempre que, entre mandos y dentro de estos, se hable de forma clara acerca de las mismas intencionalidades del trabajo y que las maneras de expresar se adapten a los perfiles 
y funciones de los coequiperos; 4) liderar y tener coequiperos no es solo cuestión de un papel sino de tener participación en la totalidad de los procesos, de lo contrario se asiste a una especialización en un fragmento de actividades que finalmente van en detrimento de los procesos y los programas; y 5) la importancia de la creatividad está propiciada por momentos de esparcimiento, en tres vías: reflexionar acerca de los controles excesivos que terminan inhibiendo la espontaneidad de las personas; diferenciar la espontaneidad de la improvisación, de no saber estar y participar según el momento y el motivo o tema, lo que da cabida a la confrontación como mecanismo deliberativo que afianza en lugar de debilitar procesos.

\section{Sobre los autores}

Faber Andrés Alzate Ortiz. Decano de la Facultad de Educación de la Corporación Universitaria Minuto de Dios (Uniminuto), Bello (Antioquia, Colombia). Es doctor en Educación con especialidad en Mediación Pedagógica de la Universidad de La Salle de Costa Rica e investigador adscrito del Grupo de Estudios e Investigaciones Educativas y Pedagógicas (Geiep). Estudió la Licenciatura en Gestión Educativa de la Universidad San Buenaventura y la Maestría en Educación de la Universidad Católica de Manizales, Colombia.

Luz Mery Chaverra Rodríguez. Docente investigadora adscrita al grupo de investigación SER de la Facultad de Ciencias de la Educación de la Universidad Católica de Oriente (UCO), Colombia. Es doctora en educación con especialidad en mediación pedagógica de la Universidad de La Salle de Costa Rica. Estudió la Licenciatura en Filosofía y Ciencias Religiosas de la Uco, la Especialización en Educación Personalizada de la Universidad Católica de Manizales, Colombia, y la Maestría en Educación de la Universidad Abierta y a Distancia de Panamá.

Elvia Patricia Arango Zuleta. Docente investigadora adscrita al grupo de investigación SER de la Facultad de Ciencias de la Educación de la Universidad Católica de Oriente (UCO). Actualmente adelanta el Doctorado en Filosofía en la Universidad Pontificia Bolivariana, de Medellín, Colombia. Estudió la Licenciatura en Educación Básica Primaria de la Universidad San Buenaventura, y en esta misma institución realizó la Especialización en Pensamiento Reflexivo, Crítico y Creativo de la Universidad San Buenaventura y la Maestría en Educación.

\section{Referencias}

Alzate, O. F. A y Jaramillo, A. A. (2015). La gestión del conocimiento un desafío para las instituciones educativas en Colombia: emergencias y tensiones desde la teoría el capital intelectual. Revista Gestión de la Educación, 5(2), 137-150. 
Barberousse, P. (2008). Fundamentos del pensamiento complejo de Edgar Morín. Revista Educare, 12(2), 95-113.

Bonnefoy, C., Cerda, G., Peine, S., Durán, M. y Ponce, Y. (2004). Gestión directiva universitaria: un instrumento para su evaluación. Revista de Psicología de la Universidad de Chile, 13(2), 63-82.

Casassus, J. (2000). Problemas de la gestión educativa en América Latina. Revista Pensamiento Educativo, 2, 282-286.

Castells, M. (2003). Modelos de universidad en la sociedad del conocimiento. En Seminario Internacional de la cátedra Unesco de E-learning de la Universitat Oberta de Catalunya [videoconferencia]. Barcelona: Universidad Abierta de Cataluña.

Chaverra R. L. M., Arango Z. E. P. y Alzate, O. F. A. (2019). Prácticas de gestión de directivos universitarios: una mirada desde los principios del paradigma emergente y un camino de oportunidades por explorar. Revista Latinoamericana de Estudios Educativos, 49(1), 67-98.

De Donini, A. y Donini, A. (2003). La gestión universitaria en el siglo xxi. Desafíos de la sociedad del conocimiento a las políticas académicas y científicas. Documento de Trabajo 107. Buenos Aires: Universidad de Belgrano. Recuperado de http:// www.ub.edu.ar/investigaciones/dt_nuevos/107_donini.pdf

Duque, E. (2009). La gestión de la universidad como elemento básico del sistema universitario: una reflexión desde la perspectiva de los stakeholders. Innovar (esp.), 25-42.

Elizalde, P. Ó. (2013). Aproximación a las ciencias de la complejidad. Revista Universidad de La Salle, (61), 45-66.

Elizondo, M. L. (2011). Competencias que debe tener un director académico universitario para la educación superior basada en competencias. Revista de Investigación Educativa, 29(1), 205-218.

Fallas, S. (2010). Un sistema de inteligencia interno para la organización inteligente. Ciencias Económicas, 28(2), 481-524.

Farfán, D. y Garzón, M. (2006). La gestión del conocimiento. Bogotá: Universidad del Rosario.

García, V., Hernández, M., Santos, C. y Fabila, A. (2009). La gestión en modalidades de programas a distancia. Estudio de caso. Apertura: Revista de Innovación Educativa, 1(1), 20-33.

Kells, H. (1997). Procesos de autoevaluación: una guía para la autoevaluación en la educación superior. Lima: Fondo Editorial de la Pontificia Universidad Católica del Perú.

Martínez, N. R. (2000). Evaluación de la gestión universitaria. Informe preparado la Comisión Nacional de Evaluación y Acreditación Universitaria. Recuperado de http://www.coneau. edu.ar/archivos/1326.pdf

Martínez, M. M. (2007[1997]). El paradigma emergente: hacia una nueva teoría de la racionalidad científica. 2. ${ }^{\mathrm{a}}$ ed. México: Trillas.

Mena, M. (2004). América Latina en busca de los nuevos modelos de educación a distancia. En M. Mena (Comp.), La educación a distancia en América Latina (26-43). Buenos Aires: La Crujía Ediciones. 
Mentado, T. y Rodríguez, J. (2011). Proceso de planificación de la formación de directivos en contextos universitarios. Modelos institucionales de formación permanente. Recuperado de http://www.ub.edu/congresice/actes/7_rev.pdf

Mintzberg, H. (1991). Mintzbergy la dirección. Madrid: Díaz de Santos.

Morin, E. (2009). Introducción al pensamiento complejo. Barcelona: Gedisa.

Palacios, G. (1996). Instituciones educativas para la calidad total. Madrid: La Muralla.

Pérez, M. y Requena, A. (2000). Evaluación institucional de la Facultad de Ciencias Políticas y Sociología de la Universidad de Granada. Revista de Educación, Cuestiones Escolares Ministerio de Educación Cultura y Deporte, 18, 420-437.

Rebolloso, E. Fernández, B., Pozo, C. y Cantón, P. (2001). Estrategias de calidad en la Universidad. Valencia: Promolibro.

Rodríguez P. J. y Aguiar P., M., V. (2015). Fortalezas y debilidades de la gestión universitaria desde la visión de los gestores. Revista Iberoamericana de Educación, 67(2), 67-82.

Roger-Ciurana, E. (2001-2002). Educación y desarrollo humano. Revista Pedagógica Tabanque, $16,9-18$.

Senge, P. (1993). La quinta disciplina. Buenos Aires: Granica.

Villarreal, E. (2005). La efectividad de la gestión escolar depende de la formación del recurso humano como factor, actor y promotor del cambio dentro de los procesos, dimensiones y políticas educativas. Revista Iberoamericana de Educación, 37(2), 2-4.

Zawacki-Richter, O. (2009). Research areas in distance education: a delphi study. International review of research in open and distance learning. Athabasca University, 10(3), 21-50. 